\title{
An Improved Digital Filtering Method Based on First Order Inertia Link
}

$$
\text { Qiang Zhou }{ }^{1, a} \text {, Lingling Wang }{ }^{2, b} \text {, Yong Liư }{ }^{3} \text {, Junwei Lei }{ }^{2}
$$

\footnotetext{
${ }^{1}$ Department of Scientific Research, Naval Aeronautical and Astronautical University, Yantai, 264001, China
}

${ }^{2}$ Department of Control, Naval Aeronautical and Astronautical University, Yantai, 264001,China

${ }^{3}$ Department of Navigation, Naval University of Engineering, Wuhan, 430033, China

aemail: zhouqiang1024@126.com, bemail: lingling0711@163.com

Keywords: Digital filter; Low-pass filter; Weighted smoothing; First order

\begin{abstract}
Digitalization of low-pass filter produced from first-order inertia link is carried out firstly in this paper. Then an improved algorithm is proposed to achieve better low-pass characteristics by smoothing the first n-part data and using the smoothed data instead of n-1 based on the first step. At last, through the detailed numerical simulation analysis, the correctness and validity of the proposed improved filter design method are verified.
\end{abstract}

\section{Introduction}

Filter is an electronic devices that passes useful frequency signal and inhibits the unwanted frequency signal, which is often used for signal processing, data transmission and interference suppression [1-3]. In the filter design, we need to first change the technical requirements of filter to that of the corresponding low-pass filter, and then design a low-pass filter to get the transfer function, and finally obtain the transfer function of the desired filter from that of the low-pass filter through the frequency transform [2-4]. This paper focuses on the low-pass filter with first-order model, the simulation of the traditional low-pass filter is carried out firstly, and weighted smoothing is used to improve the low-pass performance.

\section{Model Description of Traditional Low-pass Filter}

The traditional low-pass filter is generally designed as the following first-order system, which can be described by differential equation 1as follows [5-6].

$\dot{y}=-\frac{1}{T} y+\frac{1}{T} u$

The transfer function can also be described as following formula 2 [7].

$\frac{y}{u}=\frac{1}{T s+1}$

The following numerical equation 3 is discretized using computer programming.

$[y(n)-y(n-1)] / \Delta T=[-y(n-1)+u(n-1)] / T$

Where $\Delta T$ is the sampling interval. The formula 3 can be organized as formula 4 .

$y(n)=y(n-1)-\frac{\Delta T}{T} y(n-1)+\frac{\Delta T}{T} u(n-1)$

\section{Simulation Analysis of Traditional Low-pass Filter}

Set $T=0.1 s$, assuming that the input signal is $u=u_{a}+u_{b}, u_{a}=\sin (5 t), u_{b}=2 \sin \left(10^{5} t\right)$, and $0.2 \sin \left(10^{5} t\right)$ is the noise signal. The performance of the designed filter is verified by comparing the signal before the filter with that after the filter [8]. The simulation results are shown from figure1 to figure 3 . 


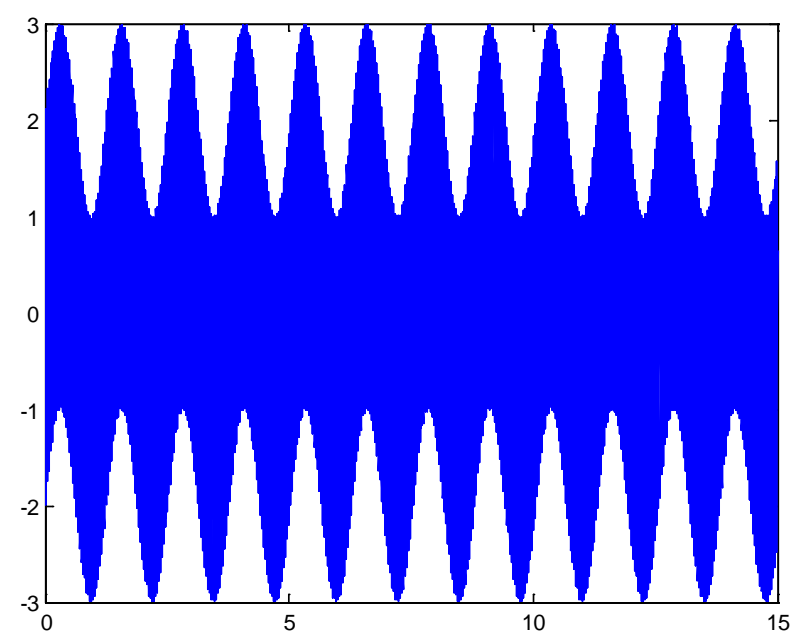

Figure 1 Source signal mixed with noise signal

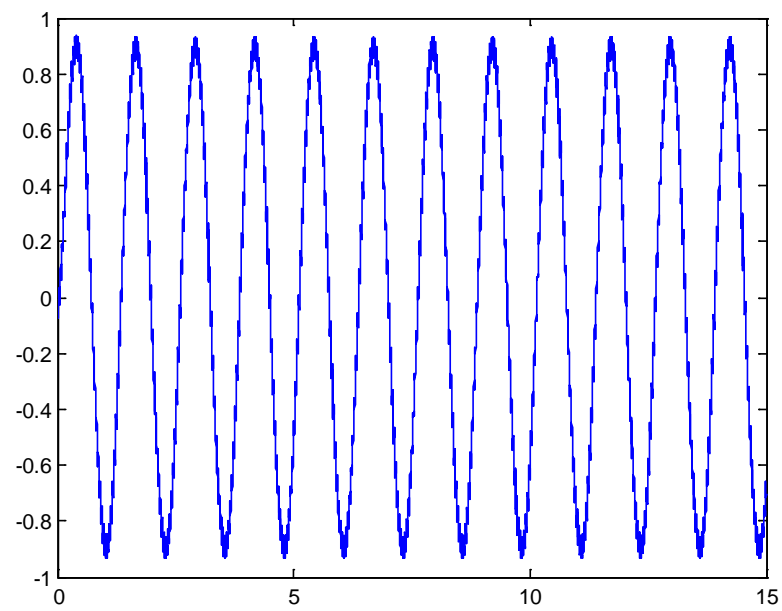

Figure 2 Filtered signal

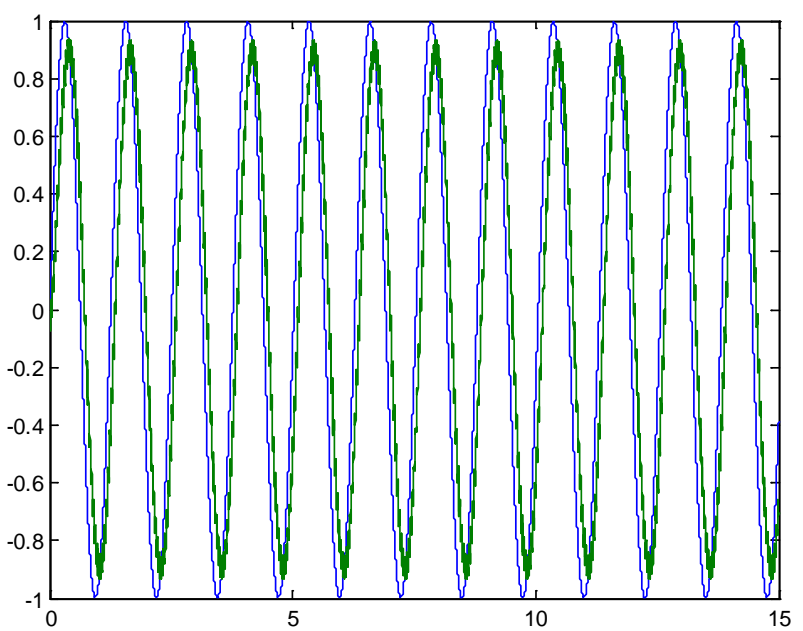

Figure 3 Comparison of filtered signal with useful signal

\section{Improvement Design by Weighted Smoothing}

The following improvement design is proposed as formula 5 . 
$y(n)=\sum_{i=0}^{j} a_{i} y(n-1-i)+\frac{\Delta T}{T} y(n-1)-\frac{\Delta T}{T} u(n-1)$

And it satisfies the formula 6.

$\sum_{i=0}^{j} a_{i}=1$

Simulation results are shown from figure 4 to figure 6 .

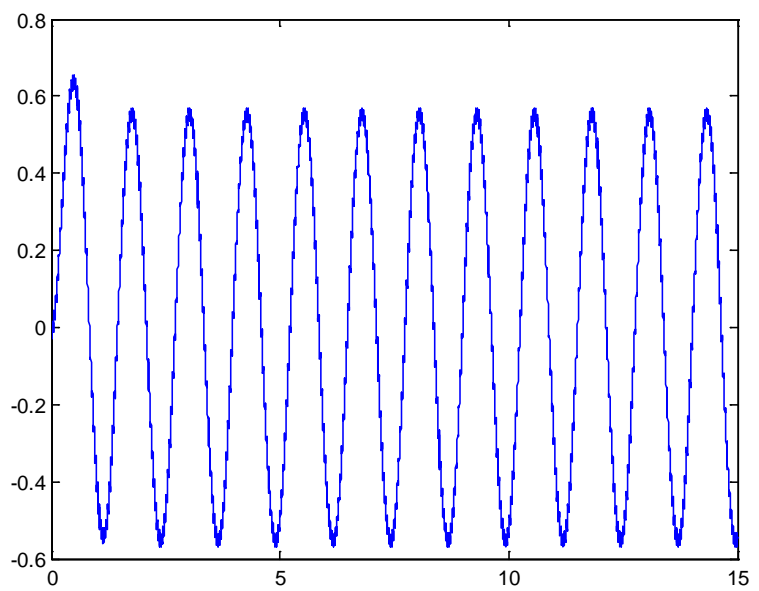

Figure 4 The signal of the improved digital filtering

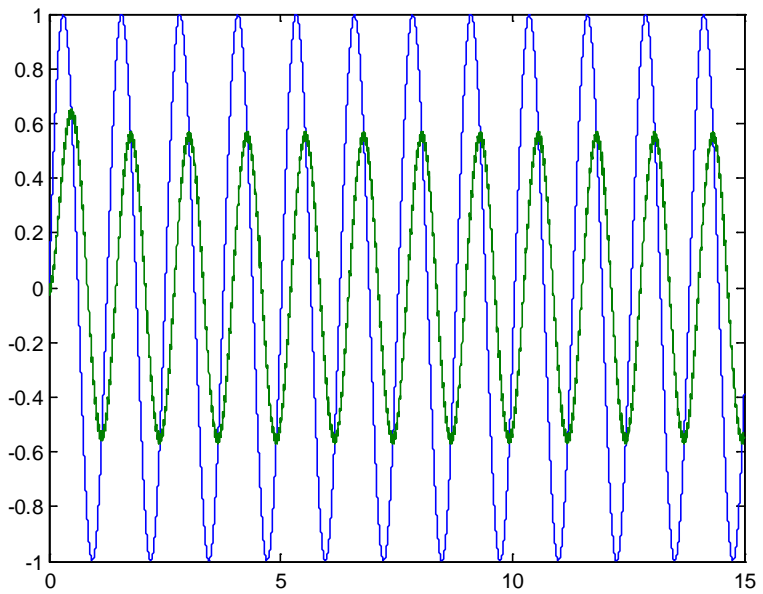

Figure 5 Comparison of the filter signal with the original signal

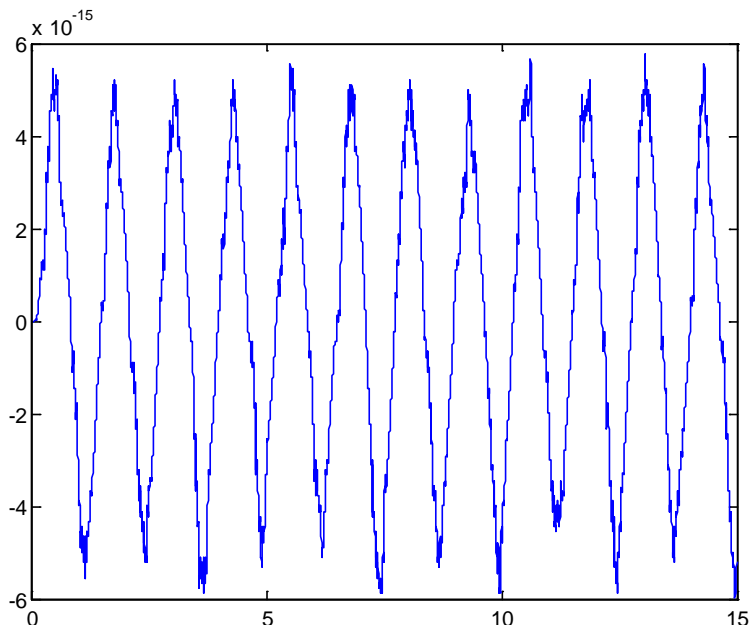

Figure 6 Error curves of two Methods

Figure 1 to Figure 3 shows the traditional filter effect and figure 4 to 6 shows the improved filter 
effect. It is obvious that figure 6 shows the difference of the two methods and the error is very small, so the method proposed in this paper can take place of the traditional method.

\section{Conclusions}

Traditional low-pass filter is designed based on first-order model in this paper. Then an improved algorithm is proposed by weighed smoothing. Simulation results show that the improve algorithm has better low-pass performance by smoothing the first n-part data and using the smoothed data instead of $n-1$.

\section{Reference}

[1] Wang Ling, Filter Research and Simulation [J], Industrial Control Computer, 2008 (7): 90-92(in Chinese).

[2] Xu Jingbo, Yuan Yibao, Liu Bo, Method of rational approximation for Gaussian low-pass filter in time domain measurement [J], Solid State Electronics Research and Development, 2011, 31 (6): 553-558(in Chinese).

[3] Zhao Yi, Digital filtering of the moving average and low-pass filter method [J]. Instrument Technology, 2001 (5): 40-40(in Chinese).

[4] Peng Yongsheng, Wang Taiyong, Low-pass filtering technology in measurement and control system [J], Precision Manufacturing and Automation, 2003 (S1): 108-110(in Chinese).

[5] Li Shengqing, Zhou Youqing, Zhu Yinghao, Harmonic current prediction method of integrated power filter based on weighted first-order local theory [J], Proceeding of the CSEE, 2004, 24 (6): 19-23(in Chinese).

[6] Lu Zhenxing, Gao Jing, Zhang Yifei, High frequency ground wave radar to eliminate the impact of first-order sea clutter on the target ranging method [J]. Telecommunications Technology, 2008, 48 (8): 43-46(in Chinese).

[7] Xia Kewen, Zhu Jun, Optimal Design of First Order Recursive Digital Filter [J]. Petroleum Equipment, 1995 (3): 164-166(in Chinese).

[8] Han Lichu et al. MATLAB Electronic Simulation and Application [M]. Beijing: National Defense Industry Press, 2003(in Chinese). 